\title{
Breaking Interaction Barriers: Monitoring Elderly in Natural Settings Exploiting Everyday Objects
}

\author{
Marina Buzzi \\ IIT-CNR, via G. Moruzzi, 156124 Pisa \\ marina.buzzi@iit.cnr.it
}

\begin{abstract}
The European population is aging steadily. As a consequence neurodegenerative pathologies are becoming widespread, impacting heavily on social costs, so it is important to support independent living as long as possible, especially in the healthy elderly.

This paper proposes an idea for advancing current monitoring technologies by breaking down the current paradigm and exploiting augmented everyday objects. Monitoring and behavior analysis can be exploited as triggers for motivating behavior changes in an elderly person. Thanks to progress in Internet of Things (IoT) and Artificial Intelligence (AI) one scenario is described to illustrate the proposed design concept. With a collaborative multidisciplinary effort, this view could be fast become a reality.
\end{abstract}

\section{Keywords}

Elderly $\cdot$ Disability $\cdot$ IoT

How to cite this book chapter:

Buzzi, M. 2020. Breaking Interaction Barriers: Monitoring Elderly in Natural Settings Exploiting Everyday Objects. In: Loizides, F., Winckler, M., Chatterjee, U., Abdelnour-Nocera, J. and Parmaxi, A. (eds.) Human Computer Interaction and Emerging Technologies: Adjunct Proceedings from the INTERACT 2019 Workshops. Pp. 13-22. Cardiff: Cardiff University Press. DOI: https://doi.org/ 10.18573/book3.b. License: CC-BY 4.0. 


\section{Introduction}

More than 25 years ago, Mark Weiser's vision greatly influenced research on ubiquitous computing, foreseeing that "the most potentially interesting, challenging and profound change implied by the ubiquitous computing era is a focus on calm." [1]. Imagining that computers would be everywhere, surrounding us in the environment, in our bodies, and in everyday objects, Weiser suggests that it is better to not be overburdened in our lives. His vision of calm technology is an image of a world that is quiet and comfortable, where we are always aware of what is happening around us, what is going to happen and what has just happened. Information would effortlessly emerge when needed and smoothly disappear from our attention [2].

In recent years, many researchers have focused on how to promote physical activity among people with cognitive impairment. Our target group includes fragile individuals such as people with disability and the elderly. A technology's invasiveness impacts on interaction. When technology is embedded and hidden within the object, its use becomes natural and simple, since no interaction is required. The idea is to modify every object which the person habitually uses in their routine activities. In the following we offer three scenarios illustrating the proposed design concept. Any technology issues still present today could be easily overcome in the near future.

Designing for the elderly as well as for persons with disability may require modifying the interaction protocol. Instead of creating new interfaces that require familiarizing oneself and becoming proficient with new objects and their apps, the design concept is to use everyday objects to collect user data, maintaining the usual interaction and not disturbing the user's routine by asking then to learn new competences.

Maintaining independence is fundamental as we age. The ability to perform personal care is one of the skills we learn early and maintain later in life; gestures become automatic day after day. This paper, in accord with Weiser's vision, proposes using ICT technology in an unobtrusive setting in order to monitor older people in their natural environment, without changing the surrounding environment or their interaction habits.

This paper is organized into four sections. After this introduction, section 2 introduces related work. In section 3 principles driving the proposed design concept are illustrated, discussing a scenario. Last, a discussion suggesting future research directions ends the paper.

\section{Related Work}

Age can degrade our physical and cognitive abilities either suddenly or progressively. Neurodegenerative pathologies such as Alzheimer's disease (AD) are receiving worldwide attention due to the enormous burden it places on families, 
health costs, and the prospect of a steadily increasing number of affected adults over the next few years [3]. IoT can help people prolong their autonomy over time. This reduces the cost of assistance, and improves the lives of the elderly and their relatives.

Dementia tests are usually a series of trials for evaluating personal abilities including memory, attention, logic, language, orientation. However these tests have usually been performed after the first signs of dementia are detected by relatives and/or clinicians. Early diagnosis could facilitate the introduction of cognitive training as well as drug therapy to control disease progress. Since events such as moving to a new home can disorient an elderly person, and accelerate or trigger the degeneration process, it is very important to keep the person in their own home, and independent as long as possible.

Technology has an increasing role in supporting people with disabilities and the elderly in everyday life. A useful overview of smart technologies and objects for assisting people with disability is offered by [4]. Sensors and RFID technology have been extensively applied to assist people with disability. Some examples include the smart cane for blind persons, and GPS-equipped shoes for persons in the early stage of Alzheimer [5].

For instance, RFID paths have been created to assist visually impaired people in walking autonomously. A smart cane equipped with an RFID reader facilitates orientation and provides information: vocal messages for directions and information about close artifacts, or services (shops, metro, etc.) [6]. Thanks to ultrasonic sensors and an obstacle detection system, a sound alert goes off if the person is leaving the main path, in order to prevent falls near the edge of the sidewalk [7]. The RFID cane reads data on the RFID tag, i.e., the tag's unique identifier, and transmits it via network to the server application. The person can also interact naturally, using their voice to specify their destination [8]. Moreover, smart shoes equipped with sensors to detect obstacles at a greater distance (more than half a meter ahead) are proposed in [9].

All this research focuses on enhancing and maintaining an individual's autonomy. This is very important for people with disability and also for the elderly. Old people wish to live in their own homes [10]. Nearly $40 \%$ of the elderly population worldwide live independently. In Northern Europe more than $90 \%$ of older persons live independently, while less than $60 \%$ in Southern Europe. In developed countries about $75 \%$ of elderly people live independently [11].

A UK survey revealed that old people worry more about losing their independence (49\%) than about dying (29\%). Similar results also emerged in a US study [12]. Moreover, losing independence was considered a bigger and more relevant issue than financial problems. In this view, one's own home is the central point for ensuring independence, health, and social inclusive activities. By exploiting technology it is possible to offer a vast range of usable services matching the needs of older people in order to combat isolation, loneliness, and related physical and mental decline $[13,14]$. Also, elderly people with neurological disability declare that their priority is to lead an independent life at home [15]. 
Literature indicates many technologies for monitoring elderly or fragile users smoothly in a domestic environment, including sensors, video cameras, RFID systems and smart devices connected to the Internet. A smart TV, which runs an operating system, is able to connect to the network, and to execute a web app. By exploiting these very familiar objects, it possible to deliver care services and support the elderly in their autonomy. The iNeighbour TV project, for instance, exploits a smart TV to promote healthcare and social interaction among seniors, their relatives, and caregivers. Delivered services include: meteo info, medication reminder, events planning, audio/video calls, read SMS and email messages. In addition, the app monitors the user by keeping track of the user's TV use. When a significant variation occurs it triggers an alert to caregivers [16].

Another example is the $\mathrm{COACH}$ project, which collects data acquired by video camera and sensors for monitoring persons who have difficulty completing everyday tasks, such as dressing or washing. Starting from collected data it is possible to infer user actions and capabilities and send appropriate messages to motivate the behavior change [17].

Progress in technology makes it possible for smart objects to interact autonomously and be able to establish relationships and shape 'social networks' of objects (Social IoT) $[14,18]$. The Socialize project for instance, exploits SIoT components including smart TVs, environmental sensors, and wearable devices (Smartwatches) to smoothly monitor and analyze user activities involving both personal and environmental parameters, under the supervision of doctors, relatives, and caregivers [19].

Recently Sawyer et al. discuss a set of challenges posed by dementia monitoring systems [20]. Specifically, Cognitive Assistive Technology (CAT) aims at monitoring and supporting people with cognitive deficits. Recent evidence suggests that spatial navigation is emerging as an important parameter in detecting preclinical signs of Alzheimer's disease: "spatial deficits, in particular related to way finding, are present in preclinical AD long before episodic memory symptoms emerge" [3].

Participative design with end users, and specifically with people with disability, can enhance the smart objects' usability and also lead to new ideas and innovative artifacts [21]. Everyday smart objects include a number of wearable devices such as smart watches, bands, and glasses. Withings has introduced a smart hairbrush to monitor the health status of one's hair. Figure 1 shows the smart brush, which thanks to the sound of hair, and the number and types of brush movements is able to evaluate the health condition of one's hair via an associated app.

However the potential of the Hair Coach that analyzes sounds could be further exploited by analyzing movements (thanks to an accelerometer and gyroscope) for monitoring elderly persons who are still in good health, in order to prolong their independent life, as discussed in the following section.

Detecting dementia at an early stage can help improve the quality of life, reducing side effects such as depression. Cognitive and drug therapy could be less effective when the damage to the brain becomes severe [20]. 


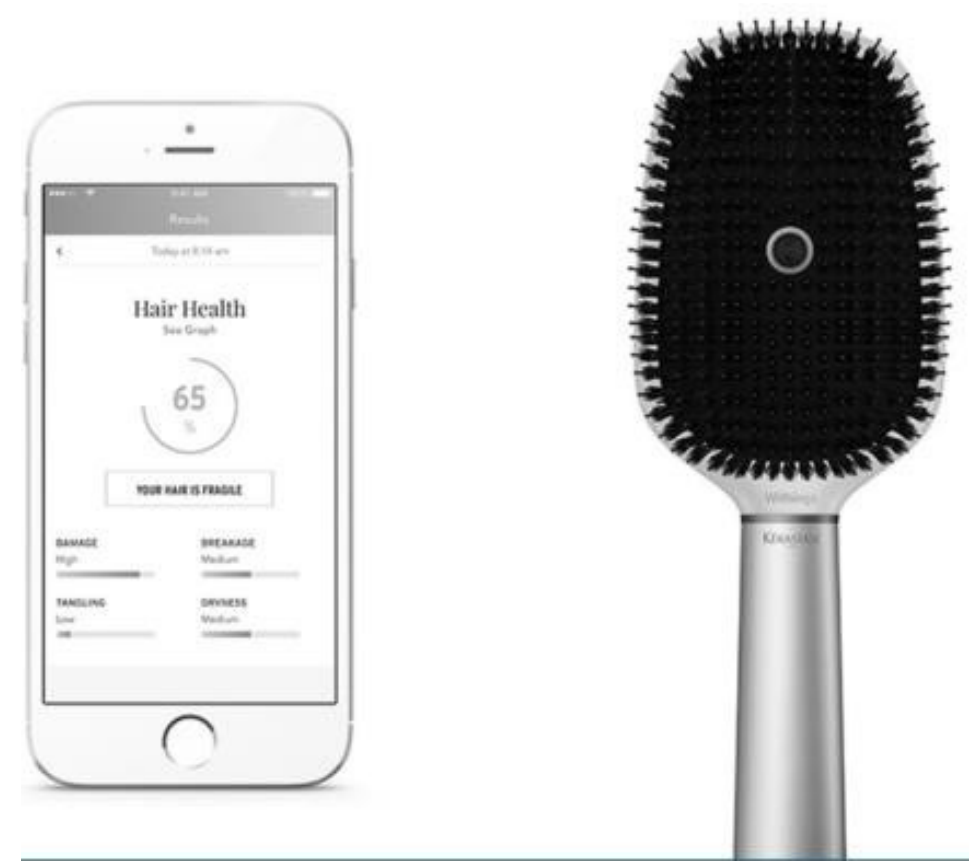

Fig. 1: Withings Kerastase smart hair brush and its Hair Health app (source: www.loreal.com).

Games such as a join-the-dots game [22] or card selection in game boards [23] can be exploited to gather assessment data. By measuring many parameters, including task completion time, latency time, mouse/pad/ finger movements it is possible to evaluate the person's attention, executive functions, perception, and motor abilities, and compare them with reference performance values [24]. Monitoring user interaction with a digital game allows one to detect cognitive deficits. However if the user feels (s)he is undergoing a form of examination, this might impact on the results [20].

Since not all older people are interested in videogames, nor are they always able to describe their condition to the doctor or perform the classic tests, observation and analysis of movements and times in their interaction with everyday objects could be an interesting alternative to investigate.

This paper aims to stimulate discussion about these relevant topics. The idea is to sensorize everyday objects and analyze a vast amount of data coming from these distributed devices, which would enable a cooperative intelligence to enhance services in many aspects of everyday life (personal care, dental hygiene, movement, psychological wellness, prevention). This hypothesis needs to be validated in order to be accepted or discarded via a prospective study, by building operative prototypes and collecting data from a significant number of users. 


\section{The Everyday Smart Object Conceptual Model: a Scenario}

An elderly woman is progressively losing some cognitive ability. Let us suppose that she can be rated in the middle of the Clinical Dementia Rating (CDR) scale $(0,0.5,1,2,3$ values), i.e., Mild 1 [25]. At this stage the person exhibits some difficulties: a) Moderate memory loss (especially for recent events). b) Moderate difficulty with time relationships; may have geographic disorientation. c) Moderate difficulty in handling problems, similarities, and differences. d) Unable to function independently in social activities (though appears normal to casual inspection). e) Mild impairment of function at home (more difficult chores, hobbies and interests abandoned). However it is important to observe that she is still able to carry out personal care but requires prompting, i.e., needs to be stimulated to perform personal care activities.

The idea is to exploit smart everyday objects to empower the individual. Smart Objects can collect and transmit data to a cloud system and thanks to artificial intelligence algorithms and natural interfaces (offering vocal and touch interaction), the processed data can provide feedback to users, through the smart object itself or via an associated smartphone app. Combining behavioral science and persuasive techniques the smart object can become a persuasive tool to keep the person's behavior positive as long as possible (in this case personal care abilities).

Let us introduce a scenario illustrating this concept. Martin is a 10-year-old boy who owns a personal smartphone. At the 80th birthday of his grandmother, Martin and his family give her a smart hairbrush and a smart toothbrush. He controls his grandmother's data via an app on his smartphone. When the app shows an alert, revealing no personal care activities have been performed by the grandmother, he sends her a video message to stimulate her to perform these activities.

Moreover, the collected data are aggregated and graphs are visible on a dashboard to summarize activities trending during the week, month and year. If a threshold is reached, an alarm is sent to her doctor who decides on the next care steps (see Fig. 2). Specifically exploiting AI and neural algorithms, it is possible to collect usage data in a preliminary training session in order to create a model of how the woman uses her hairbrush and toothbrush. Thus, over time when this model is breaking down, an alert is automatically activated, for instance:

- If the woman does not use the smart objects, an alert is sent to relatives, to check on the cause (sickness, fall, and so on).

- If the woman uses the smart objects but she is now unable to match their usage models over time (change in movements), an alert is sent to a clinician (her doctor). 

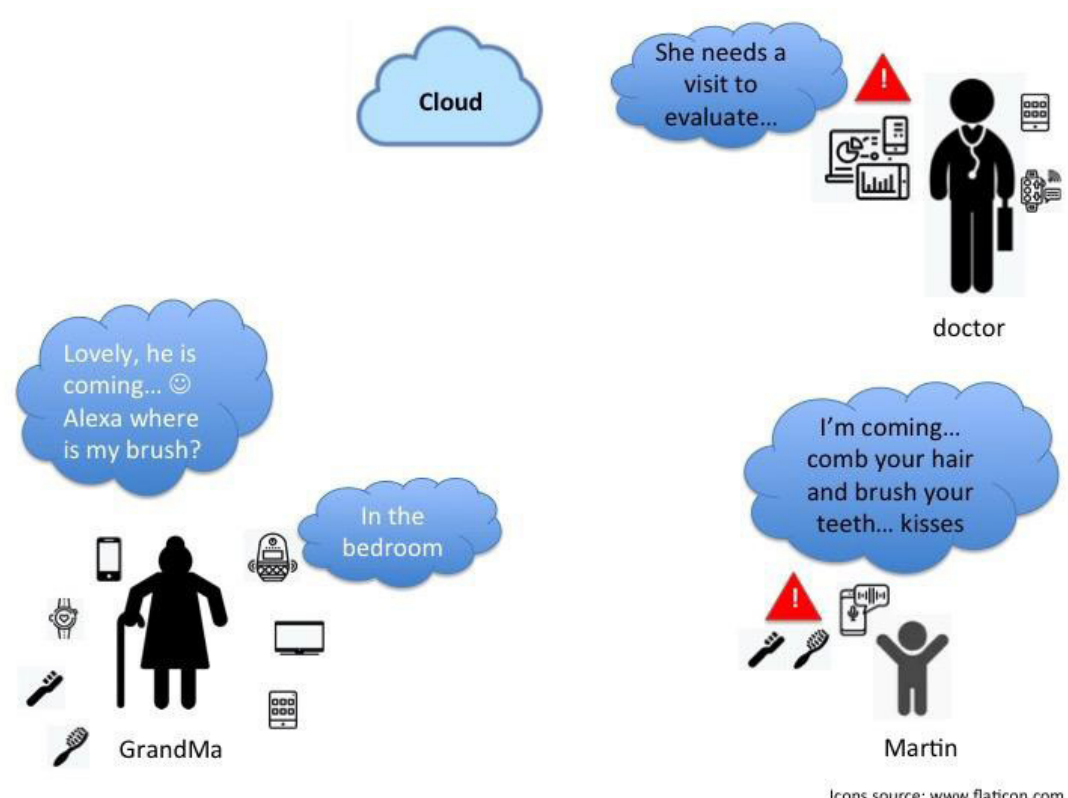

Fig. 2: Technology-enhanced care by IoT and ICT. Martin acts as his grandmother's coach. The doctor monitors main behavior changes over time.

However, bald men do not use hairbrushes, so it is crucial to design other smart objects of daily interaction. To brush one's teeth is the most frequent task performed every day. The idea is similar to the previous one: to learn the brushing patterns of the users and facilitate early detection of anomalies.

Other application fields are possible. Consider for instance children with low functioning autism, who are rigid, making dental hygiene a challenge for them. The idea is to add a micro-camera incorporated in the middle of the toothbrush to provide dental images. Lighting the oral cavity, images could be acquired and transmitted to the cloud system. Older persons can become lazy, so if the system detects some potential problem, an alert could be triggered to them and to relatives.

\section{Discussion}

Everyday objects can offer unexpected benefits, due to their frequency of use and the user's habits. For instance, they can help monitor the status of fragile users regarding their dental care.

An unexplored potential of IoT is mature for experimentation. Monitoring healthy parameters, tools and procedures while avoiding errors is an important 
emerging research field. When interfaces are dissolved inside objects the interaction is not impacted, but is maintained as usual and events or data are exploited to trigger actions and to enhance user services (context-aware intelligent services). Everyday objects can be adapted to enhance care and improve life for the elderly.

An interesting research question is whether the technology can be exploited to facilitate a diagnosis. For instance, can a change in trajectories (showing unstable movements or tremors for instance) discover early pathologies such as Parkinson's or Alzheimer's or other neurodegenerative pathologies? When something changes in these repetitive gestures it might signal body changes. These conditions could easily be detected if the software is first trained with persons with neurodegenerative diseases (to catch anomalous patterns and be able to predict a disease's onset).

This research field is extremely interesting since unobtrusive technologies might help make care more efficient, and diagnosis more precise, recognizable by data patterns. Possibly, the more data from different individuals could be analyzed, the more accurately the algorithms can learn to detect the individual's anomalous patterns.

A multidisciplinary team applying a co-design approach involving end users and professionals such as psychologists, clinicians, engineers, sociologists and HCI experts may be the key to revealing the full potential of ICT-enhanced solutions for everyday life and independence. Imagining the future of everyday objects can break down current interaction schemes and barriers, enabling monitoring of the elderly in a natural setting.

\section{References}

1. Weiser, M. Ubiquitous computing. Computer, (10), 71-72 (1993).

2. Rogers, Y. Moving on from weiser's vision of calm computing: Engaging ubicomp experiences. In International conference on Ubiquitous computing (pp. 404-421). Springer, Berlin, Heidelberg (2006).

3. Coughlan, G., Coutrot, A., Khondoker, M., Minihane, A. M., Spiers, H., \& Hornberger, M. Toward personalized cognitive diagnostics of at-geneticrisk Alzheimer's disease. Proceedings of the National Academy of Sciences, 116(19), 9285-9292 (2019).

4. Domingo, M. C. An overview of the Internet of Things for people with disabilities. Journal of Network and Computer Applications, 35(2), 584-596 (2012).

5. Wan, L., Müller, C., Randall, D., \& Wulf, V. (2016). Design of A GPS monitoring system for dementia care and its challenges in academia-industry project. ACM Transactions on Computer-Human Interaction (TOCHI), 23(5), 31.

6. Saaid, M. F., Ismail, I., \& Noor, M. Z. H. Radio frequency identification walking stick (RFIWS): A device for the blind. In 20095 th International Colloquium on Signal Processing \& Its Applications (pp. 250-253). IEEE (2009). 
7. Martin W, Dancer K, Rock K, Zeleny C, Yelamarthi K. The smart cane: an electrical engineering design project. In: Proceedings of ASEE north central section conference. Michigan, USA (2009).

8. Shiizu Y, Hirahara Y, Yanashima K, Magatani K. The development of a white cane which navigates the visually impaired. In: Proc. of the 29th annual international conference of the IEEE engineering in medicine and biology society (2007).

9. Zhang J, Lip CW, Ong SK, Nee A. A multiple sensor-based shoe-mounted user interface designed for navigation systems for the visually impaired. In: Proceedings of the fifth annual ICST wireless Internet conference (WICON). Singapore (2010).

10. Al-Shaqi, R., Mourshed, M., \& Rezgui, Y. Progress in ambient assisted systems for independent living by the elderly. SpringerPlus, 5(1), 624 (2016).

11. UN, World Population Ageing 2013. https://www.un.org/en/development /desa/population/publications/pdf/ageing/WorldPopulationAgeingReport 2013.pdf

12. Mangoni, A. A. Geriatric medicine in an aging society: up for a challenge? Frontiers in Medicine, 1, 10 (2014).

13. Shikder, S. H., Mourshed, M., \& Price, A. D. Luminaire position optimisation using radiance based simulation: a test case of a senior living room. (2010).

14. Atzori, L., Iera, A., \& Morabito, G. From" smart objects" to" social objects": The next evolutionary step of the Internet of things. IEEE Communications Magazine, 52(1), 97-105 (2014).

15. Chan, M., Campo, E., Estève, D., \& Fourniols, J. Y. Smart homes-current features and future perspectives. Maturitas, 64(2), 90-97 (2009).

16. de Abreu, J. F., Almeida, P., Afonso, J., Silva, T., \& Dias, R. Participatory design of a social TV application for senior citizens-the iNeighbour TV project. In International Conference on ENTERprise Information Systems (pp. 49-58). Springer, Berlin, Heidelberg (2011).

17. Hoey, J. Boutilier, C. People sensors decisions: Customizable and adaptive technologies for assistance in healthcare, ACM Trans. on Interactive Intelligent Systems, 2(4), pp. 1-36, 2012.

18. Atzori, L., Iera, A., Morabito, G., \& Nitti, M. The Social Internet of Things (SIoT)-when social networks meet the internet of things: Concept, architecture and network characterization. Computer networks, 56(16), 3594-3608 (2012).

19. Miori, V., Russo, D., \& Ferrucci, L. Supporting Active Aging Through A Home Automation Infrastructure for Social Internet of Things. Advances in Science, Technology and Engineering Systems Journal 3(4), 173-186 (2018).

20. Sawyer, P., Sutcliffe, A., Rayson, P., \& Bull, C. Dementia and social sustainability: challenges for software engineering. In 2015 IEEE/ACM 37th IEEE International Conference on Software Engineering (Vol. 2, pp. 527-530). IEEE (2015). 
21. Frauenberger, C., Makhaeva, J., \& Spiel, K. Designing smart objects with autistic children: Four design exposès. In Proceedings of the 2016 CHI Conference on Human Factors in Computing Systems (pp. 130-139). ACM (2016).

22. Hagler, S., Jimison, H. and Pavel, M. Assessing executive function using a computer game: Computational modeling of cognitive processes. IEEE Journal of Biomedical and Health Informatics, 18(4), pp. 1142-1152 (2014).

23. McKanna, J.A., Pavel, M. and Jimison, H. Unobtrusive monitoring of divided attention in a cognitive health coaching intervention for the elderly. Proc. American Medical Informatics Association Symposium, pp. 507-511, (2010).

24. Tong, T. and Chignell, M. Designing game-based cognitive assessments for elderly adults. Proc. 1st Int. Conf. on Gameful Design, Research, and Applications, pp. 127-130, (2013).

25. KAD, Knight Alzheimer's Disease Research Center. Clinical Dementia Rating Worksheet.https://knightadrc.wustl.edu/cdr/PDFs/CDR_Table.pdf, last accessed 2019/06/01. 